\title{
Avaliação das condições de cárie dentária de trabalhadores da Empresa Brasileira de Correios e Telégrafos em Brasília
}

\author{
Evaluation of dental caries conditions of workers \\ of the Brazilian Post and Telegraph Company in \\ Brasília
}

\section{Evaluación de las condiciones de caries dental de los trabajadores de la Empresa Brasileña de Correos y Telégrafos en Brasilia}

\author{
Talita Caroline de Sousa Oliveira ${ }^{1}$ \\ Tiago Araujo Coelho de Souza ${ }^{2}$ \\ Jorge Alberto Cordón Portillo ${ }^{2}$ \\ Lais David Amaral ${ }^{3}$

RESUMO: A cárie dentária é o principal problema de saúde bucal enfrentado no Brasil e suas sequelas podem repercutir seriamente na vida no indivíduo. O objetivo deste estudo foiavaliar a prevalência de cárie nos trabalhadores da Empresa Brasileira de Correios e Telégrafos da Diretoria Regional de Brasília, utilizando o índice CPO-D. Foram analisados 834 prontuários odontológicos de funcionários que passaram pelo exame preventivo entre 2011 e 2012 e os resultados foram comparados entre gêneros, faixas etárias e nível de escolaridade. Foram encontradas diferenças estatisticamente significantes $(\mathrm{p}<0,05)$ entre o CPO-D das diferentes faixas etárias $-11,80$ para a faixa de 23 a 34 anos; 16,55 para a faixa de 35 a 44 anos; 22,38 para a faixa de 45 a 54 anos e 23,51 para a faixa de 55 a 75 anos. Entre os níveis de escolaridade, foi encontrada diferença

1 Especialista em Odontologia em Saúde Coletiva pela Universidade de Brasília

2 Universidade de Brasília

3 Universidade Católica de Brasília

4 Secretaria de Saúde do DF 
estatisticamente significante $(\mathrm{p}<0,05)$ para o CPO-D do grupo de nível fundamental $(24,06)$ comparado com os grupos de nível médio $(19,26)$ e superior $(20,33)$. A diferenças entre o CPO-D dos grupos de nível médio e superior e dos gêneros feminino e masculino não foi estatisticamente significante $(\mathrm{p}>0,05)$.

Palavras-chave. Cárie, CPO-D, prevalência, trabalhadores.

ABSTRACT: Dental caries are the main oral health problem faced in Brazil, and its consequences may seriously affect the life of the individual. The aim of this study was to evaluate caries prevalence in the employeesof the Brazilian Post Services Company, from Brasília Regional, using DMFT index. 834 dental records of employees who had been through preventive exams between 2011 and 2012 were analyzed and the results were compared between genders, age groups and schooling levels. Statistically significant differences $(p<0,05)$ were found between the DMFT index of different age groups - 11,80 to the group from 23 to 34 years old; 16,55 to the group from 35 to 44 years old; 22,38 to the group from 45 to 54 years old; and 23,51 to the group from 55 to 75 years old. Among the groups from different schooling levels, the difference was statistically significant $(p<0,05)$ when the DMFT index of the group with Elementary School $(24,06)$ was compared with the DMFT index of the groups with Secondary $(19,26)$ and Higher Education $(20,33)$. There was found no statistically significant difference when comparing the DMFT index between the group with Secondary Education and the group with Higher Education, neither when comparing the male group's DMFT index with the female group's DMFT index.

Keywords: Caries; DMFT; prevalence; workers.

\section{INTRODUÇÃO}

A qualidade de vida é multidimensional e é afetada por diversas variáveis, como fatores externos ao indivíduo (sociais, econômicos, culturais, políticos); e fatores internos ao indivíduo (situação de saúde, valores, personalidade) ${ }^{1}$. A saúde oral, portanto, tem grande importância para a qualidade de vida, e a qualidade de vida relacionada à saúde oral (Oral Health-related quality of life - OHRQoL) tem sido alvo de estudos ${ }^{2,3}$ nos últimos anos e foi reconhecida pela organização Mundial de Saúde (OMS) como um importante segmento do Programa Global de Saúde Oral2,3. Embora processos patológicos relacionados à cavidade bucal raramente levem à mortalidade, frequentemente deixam significativas sequelas, que influenciam na saúde geral, na disposição, na auto percepção, na comunicação e na capacidade de sociabilização e de produção do indivíduo ${ }^{1,4}$.

Dentre as condições que afetam a cavidade oral, destaca-se a cárie dentária, que é o principal problema de saúde bucal enfrentado no país ${ }^{5}$. A cárie dentária é uma doença complexa, multifatorial e comportamental - sua ocorrência tem sido relacionada à contaminação pelo agente bacteriano, à susceptibilidade do hospedeiro e à dieta ${ }^{6}$; sua progressão é causada pela contínua produção de ácidos pelas bactérias, levando a perda dos minerais dentários, resultante do desequilíbrio do processo desmineralização/ remineralização ${ }^{7,8}$. Nas últimas décadas, a condição social também 
tem sido destacada como um determinante importante nos níveis de saúde bucal ${ }^{9-11}$. Tem sido observado que a privação social está associada à maior vulnerabilidade de grupos específicos ${ }^{12,13} \mathrm{e}$ que a prevalência de cárie diminui com o aumento do nível socioeconômico ${ }^{11,12}$

O acometimento pela doençacárie pode gerar várias consequências, tais como: capacidade de mastigação afetada, levando a uma alimentação precária, a alterações no peso e, consequentemente, ao prejuízo da saúde; perda de dimensão vertical; aquisição de hábitos parafuncionais; dificuldades na dicção; e efeitos estéticos com repercussões psicológicas negativas e interferência nas relações interpessoais ${ }^{3,14,24}$. Episódios de sensibilidade dolorosa também estão frequentemente associados à doença, e podem ser tão intensos a ponto de impossibilitar o indivíduo de realizar suas tarefas rotineiras, além de alterar a qualidade do sono ${ }^{14}$.

Essa importância da saúde oral para a capacidade de interação e produção do indivíduo tem influenciado também a política das empresas. Atualmente, o número de companhias que oferecem assistência à saúde tem aumentado, visto que tal prática tem se mostrado positiva não só para os funcionários, mas também às próprias instituições, em virtude da redução do absenteísmo e do aumento da capacidade de produção ${ }^{15}$.

A Empresa Brasileira de Correios e Telégrafos (ECT) é uma empresa pública, vinculada ao Ministério das Comunicações, e foi criada em 1969 pela lei 509. A execução das atividades da empresa se faz de forma descentralizada, distribuindo-se por diretorias regionais, constituídas com base no movimento financeiro, na densidade demográfica e na área da região jurisdicionada. Essas diretorias regionais são responsáveis pela operação do Serviço Postal e pela execução das atividades administrativas de rotina, observado o planejamento, a supervisão, a coordenação e o controle dos órgãos da Administração Central. O regime jurídico do pessoal da ECT é o da Consolidação das Leis de Trabalho e seus empregados são classificados na categoria profissional de comerciários. Todos os funcionários têm acesso ao Correios Saúde, benefício de Autogestão, na modalidade coletiva empresarial patrocinada, em regime de compartilhamento, que contempla atendimentos ambulatoriais, hospitalares e odontológicos, no território nacional, por meio de Ambulatório Interno da ECT e da Rede Credenciada. Na Diretoria Regional de Brasíliatrabalham 3596 funcionários.

Para a adoção de ações em saúde mais efetivas, o estudo do perfil epidemiológico dos funcionários tem sido uma importante ferramenta utilizada pelas empresas ${ }^{15,16}$. Como o combate à cárie dentária é tão importante para a manutenção da saúde oral e, consequentemente, para a saúde geral e para a qualidade de vida, índices epidemiológicos foram desenvolvidos para mensurá-la. O mais utilizado, sendo inclusive o índice recomendado pela OMS para medir e comparar a experiência de cárie nas populações, é o índice $\mathrm{CPO}^{17}$.

O índice CPO, ou índice de Ataque de Cárie, foi formulado por Klein e Palmer em 1937. Ele pode referir-se ao dente - sendo denominado CPO-D, ou à superfície dentária - CPO-S. Para o 
cálculo do CPO-D, o indivíduo é examinado e o número de dentes cariados, perdidos e obturados (restaurados) é somado. É específico para a coroa - cáries radiculares não estão incluídas - e não são necessários exames complementares ao clínico.É um índice efetivo para relatar a experiência de cárie em uma população, além de ter uma metodologia simples que pode ser compreendida internacionalmente, o que facilita a comparação de dados.O uso do índice CPO, portanto, tem sido indicado para estudos que buscam determinar o perfil de acometimento de uma determinada população ${ }^{17}$.

A correlação entre prevalência de cárie e grupos com características distintas também tem sido estudada em diferentes pesquisaspor meio do CPO-D ${ }^{17-27}$. Em relação ao gênero, alguns estudos relatam uma maior prevalência no gênero feminino e associam o fato à erupção precoce dos dentes nesse grupo ${ }^{18,19}$. Sales-Peres et al. ${ }^{20}$, no entanto, encontraram maior prevalência no gênero masculino em virtude da maior conscientização ao auto-cuidado e melhor e mais frequente controle mecânico da placa por parte das mulheres. Por outro lado, em outros estudos de Peres et $\mathrm{al} .{ }^{21} \mathrm{e}$ de Feysleben et $a l .^{22}$ não foram encontradas diferenças estatisticamente significantes entre os gêneros. Em relação à idade, visto que a cárie dentária tem caráter cumulativo, indivíduos mais velhos apresentam valores mais altos de $\mathrm{CPO}-\mathrm{D}^{17}$.Em adição, estudos mostram que existe relação entre a prevalência de cárie indicada pelo CPO-D e a renda familiar e os níveis de escolaridade e inserção social ${ }^{23-27}$.

Tendo emvista estas considerações, tem sido sugerido na literatura que o levantamento epidemiológico da saúde bucal de uma população, utilizando-se o CPO-D, é um importante instrumento para a avaliação da necessidade de tratamento e das estratégias preventivas que devem ser adotadas ${ }^{16}$, de modo a elaborar-se um planejamento que seja focado nas reais necessidades, oferecendo tratamento efetivamente resolutivo ${ }^{28}$.

O presente estudo teve como objetivo geral conhecer a situação da saúde bucal de trabalhadores da Empresa Brasileira de Correios e Telégrafos na Diretoria Regional de Brasília e avaliar, por meio do CPO-D, a prevalência de cárie geral, segundo gênero, faixa etária e nível de escolaridade.

\section{MATERIAL E MÉTODOS}

No presente estudo, foram avaliados os prontuários odontológicos dos funcionários da Empresa Brasileira de Correios e Telégrafos da Diretoria Regional de Brasília (ECT DR-BSB) que passaram pelo exame preventivo entre 2011 e 2012. Todos os funcionários tinham acesso ao benefício "Correios Saúde”. Inicialmente, haviam 2187 prontuários. Foram excluídos prontuários com dados incompletos e prontuários que se referiam a um mesmo indivíduo (tendo sido analisado apenas o mais recente), restando 834prontuários.

Os exames odontológicos preventivos dos trabalhadores da Empresa Brasileira de Correios e Telégrafos são realizados em cada funcionário uma vez a cada dois anos, para aqueles que têm menosde quarenta anos, e anualmente, para os demais. Os exames foram realizados em consultórios 
odontológicos, por sete cirurgiões-dentistas contratados pela empresa, em ambulatórios próprios dos Correios, no Distrito Federal, nos seguintes setores: Setor Hoteleiro Sul, Setor de Indústrias Automobilísticas e Gama. Durante o exame clínico, os dentistas preenchiam uma ficha clínica e um odontograma. As superfícies restauradas foram marcadas de azul; superfícies cariadas, marcadas de vermelho; dentes perdidos foram assinaladas com a letra " $X$ "; se a superfície apresentava-se hígida, nenhuma marcação foi feita

As fichas foram entregues a um funcionário responsável por registrar os dados em uma planilha no programa Excel. Na planilha, havia o registro da matrícula, da data de nascimento, do nível de escolaridade, do cargo, do gênero e do registro da condição odontológica de cada funcionário, por dente. As células de dentes hígidos foram preenchidas com a letra " $\mathrm{H}$ "; para dentes com restauração foi usada a letra "O"; células de dentes com lesão de cárie (restaurados ou não) foram preenchidas com a letra "C"; quando o dente havia sido perdido a marcação foi feita com a letra "A".

A partir dos dados, foi calculado o CPO-Ddos funcionários examinados. Em adição, foi realizada uma estratificação deste CPO-D para os componentes cariado, perdido e obturado. Também foram calculados e estratificados os índices de CPO-D para os seguintes grupos: gênero masculino e feminino; nível de escolaridade (fundamental, médio e superior) e diferentes faixas etárias (23 - 34 anos; 34 - 44 anos; 45 - 54 anos; 55 - 75 anos). A análise de variância (ANOVA) foi aplicada para os resultados obtidos.

O uso do Termo de Consentimento Livre e Esclarecido e a submissão ao Comitê de Ética não se aplicaram a esse trabalho, pois a pesquisa foi realizada com dados históricos de prontuários de pacientes, por uma funcionária dos Correios, com autorização da Gerência de Saúde da Diretoria Regional de Brasília.

\section{RESULTADOS}

Foram analisados os prontuários de 834 funcionários da ECT DR-BSB que realizaram os exames odontológicos preventivos entre 2011 e 2012. O CPO-D foi igual a 19,66. O índice do componente $\mathrm{C}$ foi o menor, sendo igual a 0,73 . Já o valor do componente $\mathrm{P}$ foi igual a 8,57 e o componente $\mathrm{O}$ foi o mais representativo, com média de 10,36.

Em relação ao gênero, 584 funcionários eram do gênero masculino e 250 do gênero feminino. O CPO-D para os homens foi igual a 19,52, enquanto os das mulheres foi de 19,97. Essa diferença não foi estatisticamente significante $(\mathrm{p}=0,412)$. A Tabela 1 mostra os resultados. 
TABELA 1 - Número de funcionários examinados por gênero e CPO-D

\begin{tabular}{|c|c|c|}
\cline { 2 - 3 } \multicolumn{1}{c|}{} & Masculino & Feminino \\
\hline CPO-D & 19,52 & 19,97 \\
\hline Número de funcionários & 584 & 250 \\
\hline
\end{tabular}

Fonte: ECT, DR-BSB, 2011-2012

Para o CPO-D das mulheres, o componente mais significativo foi o componente $\mathrm{O}$ com índice de 11,75, seguido pelo componente P com 7,61 e pelo componente $C$, igual a 0,61. Em relação ao CPO-D dos homens, o componente O também apresentou o maior índice, com o valor de 9,77 , seguido pelo componente $\mathrm{P}$, igual a 8,98 , e pelo componente $\mathrm{C}$, igual a 0,78 . Esses valores estão demonstrados na Tabela 2 e no Gráfico 1.

TABELA 2 - Estratificação do CPO-D para os gêneros masculino e feminino

\begin{tabular}{|c|c|c|}
\cline { 2 - 3 } \multicolumn{1}{c|}{} & \multicolumn{2}{c|}{ Gênero } \\
\cline { 2 - 3 } \multicolumn{1}{c|}{} & Masculino & Feminino \\
\hline Componente C & 0,78 & 0,61 \\
\hline Componente $\mathbf{P}$ & 8,98 & 7,61 \\
\hline Componente $\mathbf{O}$ & 9,77 & 11,75 \\
\hline
\end{tabular}

Fonte: ECT, DR-BSB, 2011-2012

GRÁFICO 1 - Estratificação do CPO-D para os gêneros masculino e feminino 15

10

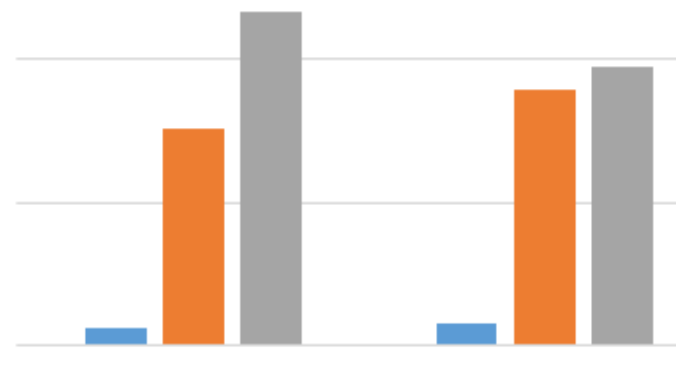

Gênerofemino Gêneromasculino

\section{$\mathrm{C} \square \mathrm{P} \square \mathrm{O}$}

Fonte: ECT, DR-BSB, 2011-2012

Na divisão por faixa etária, O CPO-D para a faixa de 23 a 34 anos foi de 11,80, para um total de 136 indivíduos. Para a faixa de 35 a 44 anos, com 179 funcionários, o CPO-D encontrado foi igual a 16,55. Para a faixa de 45 a 54 anos, o valor do CPO-D foi de 22,38 e o grupo foi composto por 334 pessoas. Finalmente, para a faixa de 55 a 75 anos, composta por 185 funcionários, o CPO-D 
foi de 23,51. Os valores diferiram de maneira estatisticamente significante $(p<0,000)$. A Tabela 3 e o Gráfico 2 mostram a comparação por faixa etária.

TABELA 3 - Estratificação do CPO-D de acordo com a faixa etária

\begin{tabular}{|c|c|c|c|c|c|}
\hline $\begin{array}{c}\text { Faixa } \\
\text { etária }\end{array}$ & CPO-D & $\begin{array}{c}\text { Componente } \\
\mathbf{C}\end{array}$ & Componente P & $\begin{array}{c}\text { Componente } \\
\mathbf{O}\end{array}$ & $\begin{array}{c}\text { Número de } \\
\text { funcionários } \\
\text { examinados }\end{array}$ \\
\hline $23-34$ & 11,80 & 0,83 & 2,91 & 8,06 & 136 \\
\hline $35-44$ & 16,55 & 0,67 & 4,62 & 11,26 & 179 \\
\hline $45-54$ & 22,38 & 40,75 & 10,37 & 11,26 & 334 \\
\hline $55-75$ & 23,51 & 0,66 & 13,29 & 9,58 & 185 \\
\hline
\end{tabular}

Fonte: ECT, DR-BSB, 2011-2012

GRÁFICO 2 - Estratificação do CPO-D de acordo com a faixa etária

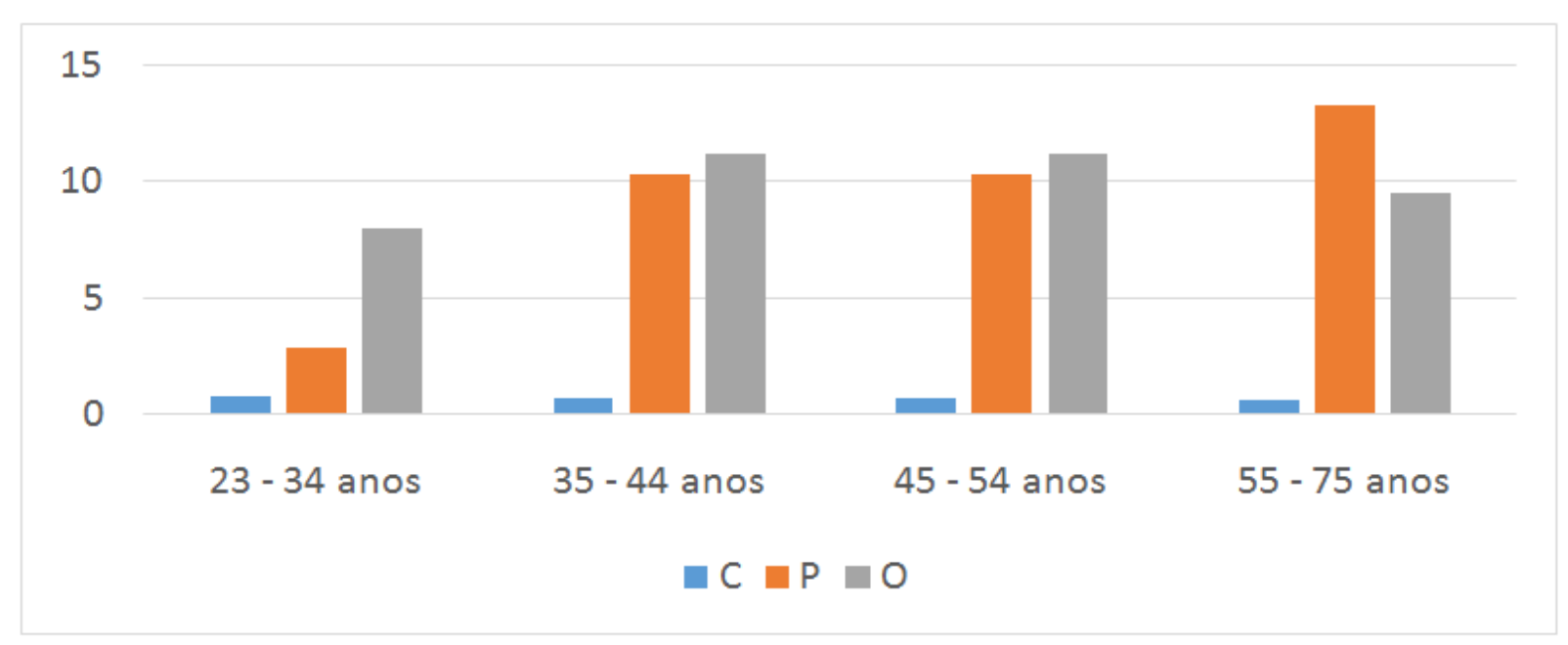

Fonte: ECT, DR-BSB, 2011-2012

Quando foram analisados os dados relativos ao nível de escolaridade, o CPO-D do grupo com nível superior foi igual a 20,33, enquanto o de nível médio foi igual a 19,26. Esses valores não diferiram de maneira estatisticamente significante $(p=0,170)$. Já o de nível fundamental foi 24,06 . A diferença do CPO-D do nível fundamental para os níveis médio e superior foi estatisticamente significante $(\mathrm{p}<0,000$ e $\mathrm{p}=0,001$, respectivamente $)$.

Para o grupo de nível fundamental o componente com maior prevalência foi $\mathrm{P}$, com 15,13. O componente $\mathrm{O}$ teve índice igual a 8,35 e o componente $\mathrm{C}$ igual a 0,58 . $\mathrm{Na}$ análise dos dados do grupo de nível médio, constatou-se índice de 0,73 para $\mathrm{C}, 10,22$ para $\mathrm{O}$ e 8,32 para $\mathrm{P}$. No grupo de nível superior, o componente $\mathrm{O}$ obteve média de 12,56 , o grupo $\mathrm{P}$ de 6,84 e o grupo $\mathrm{C}$ de 0,8 . A Tabela 4 e o Gráfico 3 mostram os resultados. 
Tabela 4 - Estratificação do CPO-D de acordo com o nível de escolaridade

\begin{tabular}{|c|c|c|c|c|c|}
\hline $\begin{array}{c}\text { Nível de } \\
\text { escolaridade }\end{array}$ & CPO-D & $\begin{array}{c}\text { Componente } \\
\mathbf{C}\end{array}$ & Componente P & $\begin{array}{c}\text { Componente } \\
\mathbf{O}\end{array}$ & $\begin{array}{c}\text { Número de } \\
\text { funcionários } \\
\text { examinados }\end{array}$ \\
\hline Fundamental & 24,06 & 0,58 & 15,13 & 8,35 & 48 \\
\hline Médio & 19,26 & 0,73 & 8,32 & 10,22 & 690 \\
\hline Superior & 20,33 & 0,80 & 7,10 & 12,43 & 96 \\
\hline
\end{tabular}

Fonte: ECT, DR-BSB, 2011-2012

GRÁFICO 3 - Estratificação do CPO-D de acordo com o nível de escolaridade

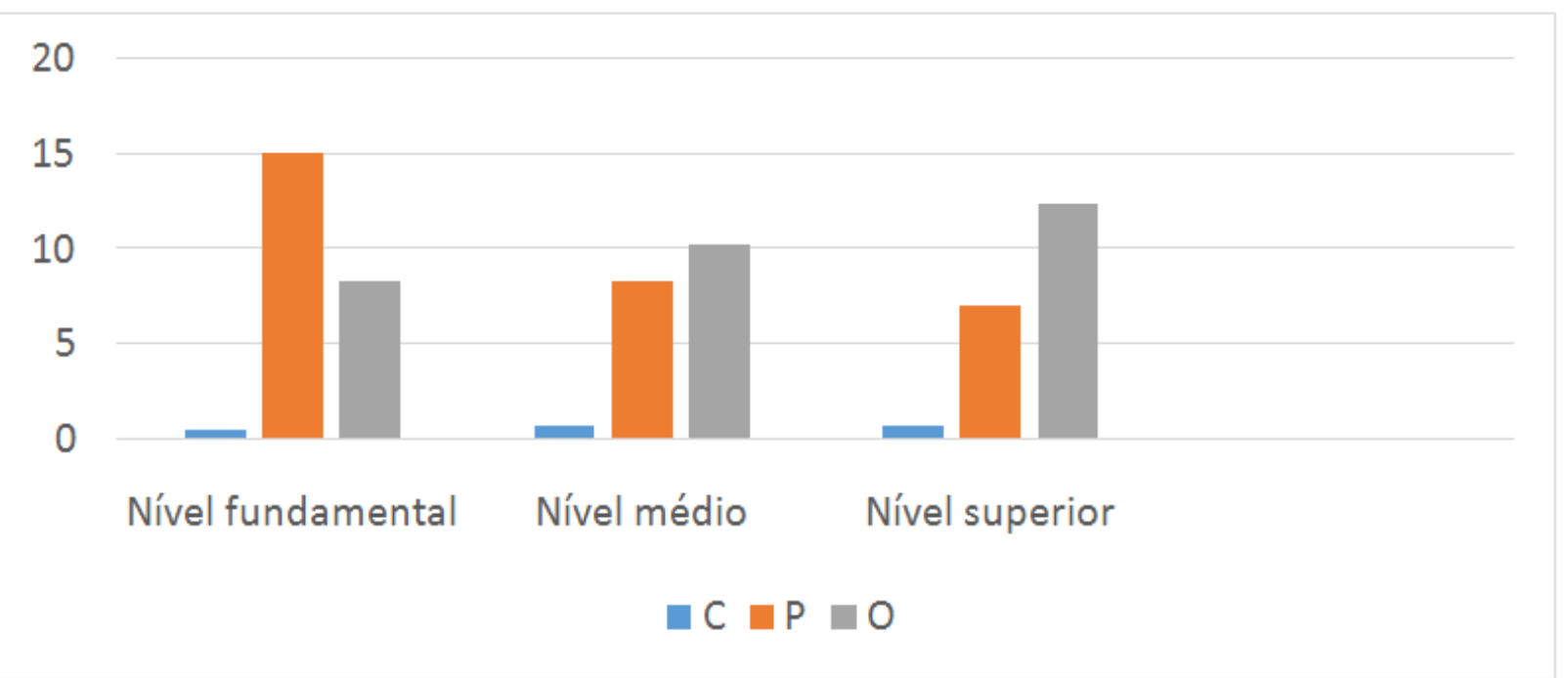

Fonte: ECT, DR-BSB, 2011-2012

\section{DISCUSSÃO}

Ao analisar o CPO-D do total dos funcionários, nota-se que a maior parte do índice $(96,28 \%)$ refere-se aos dentes perdidos e obturados, apenas 3,72\%a dentes cariados. A análise destes dados indica que os exames preventivos, nos quais os pacientes são orientados sobre sua saúde oral, e o acesso a tratamento odontológico, parecem auxiliar na manutenção da saúde dos trabalhadores.

O estudo possui limitações, pois foi baseado em exames secundários, por meio da análise de prontuários preenchidos por funcionários da empresa, para propósitos diversos da realização desta pesquisa. É importante observar que, no preenchimento dos prontuários, não foi distinguido se os dentes ausentes foram perdidos em decorrência da cárie dentária ou de outros fatores (trauma, razões ortodônticas, agenesias dentárias), o que tende a sobrestimar o CPO-D. Também deve ser considerado o viés da amostra, que é composta majoritariamente por indivíduos do gênero masculino e que trabalham como carteiros.

Em relação ao gênero, observa-se que o número de homens que trabalham na empresa é muito maior que o número de mulheres. Tal constatação vai ao encontro das informações do censo do Instituto Brasileiro de Geografia e Estatística (IBGE) de 2010, que indicam que o nível de ocupação geral é maior entre os indivíduos do gênero masculino ${ }^{29}$. Assim como na análise do CPO-D geral, 
nota-se que, tanto para os homens quanto para as mulheres, o maior componente do CPO-D é o componente $\mathrm{O}$, seguido pelo componente $\mathrm{P}$. Em ambos, portanto, é constatado que os componentes "obturado" e "perdido" formam a maior parte do índice.A média do CPO-D desses grupos não apresentou diferença estatisticamente significante, assim como nos estudos de Peres et al.(2008) ${ }^{21}$ e de Feysleben et al. $(2000)^{22}$. Mais estudos são necessários para esclarecer se há diferenças de CPO-D entre gêneros.

$\mathrm{Na}$ análise por faixas etárias, observa-se o caráter cumulativo da doença cárie. Assim como tem sido constatado na literatura ${ }^{17,25}$, observou-se o aumento do CPO-D com a progressão da idade. Em relação ao CPO-D da população em geral, relatado na Pesquisa Nacional de Saúde Bucal realizada em 2010 (SB-Brasil 2010) , podemos notar que o CPO-D dos funcionários de 35 a 44 anos da ECT $(16,5)$ é semelhante ao nacional $(16,3)$. Entretanto, na análise por componentes, observa-se que os componentes cariado e perdido têm influência maior no índice nacional $(\mathrm{C}=1,9 ; \mathrm{P}=7,3$ e $\mathrm{O}=7,1)$. Portanto, o acesso a emprego, plano de saúde e orientações periódicas pareceram ser importantes para a saúde bucal dos empregados da ECT DR-BSB.

$\mathrm{Na}$ análise por escolaridade, não houve diferença estatisticamente significante entre os níveis médio e superior. No entanto, nota-se que o maior CPO-D está no grupo de ensino fundamental, e essa diferença foi estatisticamente significante para os outros dois grupos $(p<0,05)$. Tal observação está de acordo com diversos estudos ${ }^{23-27,25}$ que indicam que o nível socioeconômico tem influência direta na saúde bucal e, consequentemente, no CPO-D. Em relação a esses dados, é interessante observar que o plano de saúde e o acesso ao tratamento nos ambulatórios da empresa para todos os funcionários é o mesmo, com acesso aos mesmos procedimentos e à mesma rede credenciada, além de todos participarem de programas preventivos. Portanto, para que hajam avanços nas condições de saúde bucal da população, o acesso aos serviços de saúde é essencial, mas não é suficiente. São necessárias medidas integradas, que abranjam educação, habitação e condições de trabalho e aumento de renda.

\section{CONCLUSÕES}

Embora o CPO-D dos trabalhadores da ECT tenha sido semelhante ao da população brasileira, para os funcionários da empresa foi menor a participação dos componentes cariados e perdidos. Nota-se a importância do emprego, do acesso ao tratamento odontológico e da orientação sobre saúde para a redução da prevalência de cárie.

O nível de educação também exerceu influência sobre o CPO-D dos empregados, embora o acesso aos serviços de saúde fosse igual a todos os grupos. Conclui-se que conhecimento do perfil de saúde bucal de uma determinada população é importante para que o planejamento das ações de saúde seja focado nas reais necessidades e, consequentemente, mais efetivo. Essas ações, em adição, não devem focar apenas a assistência à saúde, mas a qualidade de vida em geral, englobando educação, condições de trabalho e nível de renda.

\section{REFERÊNCIAS BIBLIOGRÁFICAS}

1. Cohen-Carneiro F, Souza-Santos R, Rebelo MA. Cien Saude Colet. Qualidade de vida 
relacionada à saúde bucal: contribuição dos fatores sociais. 2011;16 Suppl 1:1007-15

2. Sischo L, Broder HL. J Dent Res. Oralhealth-relatedquality of life: what, why, how, and future implications. 2011;90(11):1264-70.

3. Naito M, Yuasa H, Nomura Y, Nakayama T, Hamajima N, Hanada N. J Oral Sci.Oral healthstatus and health-relatedquality of life: a systematic review. 2006;48(1):1-7

4. de Lima DC, Saliba NA, Garbin AJ, Fernandes LA, Garbin CA. Cien Saude Colet. A importância da saúde bucal na ótica de pacientes hospitalizados.2011;16 Suppl 1:1173-80.

5. Ministério da saúde. Secretaria de Política de Saúde. Departamento de Atenção Básica. Área Técnica de Saúde Bucal.Projeto SB 2010. Condições de Saúde Bucal da População Brasileira no ano 2010.2011.

6. Al-Dajani M, Limeback H. J Calif Dent Assoc. Emerging science in the dietary control and prevention of dental caries.2012;40(10):799-804

7. Lussi A, Hellwig E, Klimek.Schweiz Monatsschr Zahnmed.Fluorides - mode of action and recommendations for use. 2012;122(11):1030-42

8. Pitts N, Duckworth RM, Marsh P, Mutti B, Parnell C, Zero D. Br Dent J. Post-brushing rinsing for the control of dental caries: exploration of the available evidence to establish what advice we should give our patients. 2012; 13;212(7):315-20

9. Baldani MH, Narvai PC, Antunes JLF. Cárie dentária e condições sócio-econômicas no Estado do Paraná, Brasil, 1996. Cad. Saúde Pública. 2002;18(3):755-763

10. Loretto NRM, Seixas ZA, Jardim M C, Brito RL. Rev ABO Nacional. Cárie dentária no Brasil: Alguns aspectos sociais, políticos e econômicos. 2000;8:45-49

11. Martins MD, Araújo RGD, Veloso NF. Jornal Brasileiro de Odontopediatria e Odontologia do Bebê. Avaliação das necessidades de tratamento odontológico de crianças de baixa renda. 1999;2:132-136

12. Jones CM, Worthington H. Journal of Dentistry. Water fluoridation, poverty and tooth decay in 12-year-old children. 2000; 28:389-393

13. Peres KGA, Bastos JRM, Latorre MRDO. Rev Saúde Pública. Severidade de cárie em crianças e relação com aspectos sociais e comportamentais. 2000;34:402-408

14. Bönecker M, Abanto J, Tello G, Oliveira LB. Braz Oral Res. Impact of dental caries on preschoolchildren'squality of life: an update. 2012;26 Suppl 1:103-7.

15. Costa Filho LC, Duncan BB, Polanczyk CA, Sória ML, Habekost AP, Costa CC. Cad Saude Publica. Análise do impacto econômico-assistencial de duas abordagens para redução de custos em um plano odontológico de autogestão.2008;24(5):1071-81 
16. Sheiham A, Sabbah W. Caries Res. Using universalpatterns of caries for planning and evaluating dental care. 2010;44(2):141-50

17. Pinto, V. G. Saúde bucal coletiva. São Paulo, SP: Livraria Santos, 2013 - $6^{\text {a }}$ edição.

18. Moreira PV, Rosenblatt A, Passos IA.Cien Saude Colet. Prevalência de cárie em adolescentes de escolas públicas e privadas na cidade de João Pessoa, Paraíba, Brasil. 2007;12(5):1229-36

19. Vieira SCM. Cárie dentária em primeiros molares permanentes: um estudo epidemiológico em escolares de 7 a 12 anos de idade na cidade do Recife, Brasil [tese]. Camaragibe (PE): Faculdade de Odontologia de Pernambuco; 2002.

20. Sales-Peres SH, Bastos JR. Cad Saude Publica. Perfil epidemiológico de cárie dentária em crianças de 12 anos de idade, residentes em cidades fluoretadas e não fluoretadas, na Região Centro-Oeste do Estado de São Paulo, Brasil.2002;18(5):1281-8

21. Peres SH, de Carvalho FS, de Carvalho CP, Bastos JR, Lauris JR.Cien Saude Colet. Polarização da cárie dentária em adolescentes, na região sudoeste do Estado de São Paulo, Brasil. 2008;13 Suppl 2:2155-62

22. Freysleben GR, Peres MAA, Marcenes W. Rev. Saúde Pública.Prevalência de cárie e CPOD médio em escolares de doze e treze anos de idade nos anos de 1971 e 1997, região Sul, Brasil. 2000;34(3):304-308.

23. Baldani MH, Vasconcelos AG, Antunes JL. Cad Saude Publica.Associação do índice CPO-D com indicadores sócio-econômicos e de provisão de serviços odontológicos no Estado do Paraná, Brasil. 2004;20(1):143-52

24. Peres KGA, Bastos JRM, Latorre MRDO. Severidade de cárie em crianças e relação com aspectos sociais e comportamentais. Rev Saúde Pública. 2000;34:402-8

25. Nadanovsky P. O declínio da cárie. In: Pinto VG, organizador. Saúde bucal coletiva. $4^{\mathrm{a}}$ Ed. São Paulo: Santos; 2000. p. 341-51

26. Baldani MH, Narvai PC, Antunes JLF. Cad Saúde Pública. Cárie dentária e condições socioeconômicas no Estado do Paraná, Brasil, 1996. 2002;18:755-63

27. Peres MA, Peres KG, Traebert J, Zabot NE, Lacerda JT. J Adolesc Health. Prevalence and severity of dental caries are associated with the worst socioeconomicconditions: a Brazilian cross-sectional study among 18-year-old males. 2005;37(2):103-9

28. Pinto VG, Lima MOP. Bib Vir MS. Estudo epidemiológico de saúde bucal em trabalhadores da indústria. 2006;CDU 616.314-058.243.2(81)

29. Instituto Brasileiro de Geografia e Estatística. Censo 2010 [acesso em 02 jan 2012].

Disponível em: http://censo2010.ibge.gov.br/noticias-censo?view=noticia\&id=1\&idnoticia=2296

ISSN 1982-8829 Tempus, actas de saúde colet, Brasília, 14(1), 197-208 mar, 2020. Epub Mai/2020 
$\& \mathrm{t}=$ censo-2010-mulheres-sao-mais-instruidas-que-homens-ampliam-nivel-ocupacao

30. Oliva JM, Lima Abraão, MRA. O impacto na qualidade de saúde bucal dos trabalhadores dos Correios atendidos no programa Preveniu, Sorriu. Monografia [Especialização em odontologia do Trabalho] - Sindicato dos Odontologistas do DF; 2008

31. Rihs LB, da Silva DD, de Sousa Mda L. J Appl Oral Sci. Dental caries and tooth loss in adults in a Brazilian southeastern state.2009;17(5):392-6.

32. Sheiham A, Steele JG, Marcenes W, Tsakos G, Finch S, Walls AW. Community Dent Oral Epidemiol. Prevalence of impacts of dental and oral disorders and their effects on eating among older people; a national survey in Great Britain. 2001;29(3):195-203.

33. Patrick DL, Chiang YP. Med Care. Measurement of healthoutcomes in treatment effectiveness evaluations: conceptual and methodological challenges.2000 Sep;38(9 Suppl):II14-25

34. Ministério da saúde. Secretaria de Política de Saúde. Departamento de Atenção Básica. Área Técnica de Saúde Bucal. Condições de Saúde Bucal da População Brasileira no ano 2003. Projeto SB 2003.2004.

35. Tomita NE, Cordeiro R, Mendonça J, Senger V, Lopes ES.Rev FOB. Saúde bucal dos trabalhadores de uma indústria alimentícia do Centro-Oeste paulista. 1999; 7(1):67-71

36. Carnut L.J Manag Prim Health Care. Planning and programming of health activities: concepts, importance and their influences on the organization of oral health services. 2012;3(1):53-61.

37. Ministério da Saude. Saúde bucal. Caderno de Atenção Básica nº17. $1^{a}$ edição - 2008

38. World Health Organization. Oral health information systems [acesso em 03 mai 2012]. Disponível em: http://www.who.int/oral_health/action/information/surveillance/en/index.html

39. Maia EG, Cavalcante GMS, Granville-Garcia AF, Lucas RSCC, Cavalcanti AL, d'Avila S. Arq Odontol BH. Condição de saúde bucal de trabalhadores atendidos no Núcleo de saúde Ocupacional de Campina Grande - PB. 2012; 48(1):32-39

40. Cypriano S, Sousa MLR, Wada RS. Rev. Saúde Pública. Avaliação de índices CPOD simplificados em levantamentos epidemiológicos de cárie dentária.2005;39(2):285-292A

Artigo apresentado em outubro de 2029 Artigo aprovado em janeiro de 2020 Artigo publicado em julho de 2020 